\title{
VLBA Polarimetry of GPS Quasar OQ172
}

\author{
Y. Liu, D. R. Jiang, \& M. F. Gu \\ Key Laboratory for Research in Galaxies and Cosmology, Shanghai Astronomical Observatory, \\ Chinese Academy of Sciences, \\ 80 Nandan Road, Shanghai 200030, China \\ email: yliu@shao.ac.cn
}

\begin{abstract}
We present a study of the VLBA polarimetry of GHz-Peaked Spectrum (GPS) quasar OQ172. GPS quasar OQ172 is one of the source with the extremely highest rest-frame rotation measure $\left(\mathrm{RM}>20,000 \mathrm{rad} / \mathrm{m}^{2}\right)$. By analyzed our of VLBA polarimetry observation, we found the Rotation Measure on C \& X band would be well fitted by linear relationship of the square of $\lambda^{2}$. Combined other evidences of physical environment in the core of OQ172, we suggest that this source might be a young AGN.
\end{abstract}

Keywords. Galaxies: jet - galaxies: nuclei - quasars: individual: OQ172

\section{Introduction}

GHz-Peaked Spectrum (GPS) radio sources are characterized by their compact size $(\mathrm{L}<\sim 100 \mathrm{pc})$ and convex spectrum peaked at $\sim 0.5-10 \mathrm{GHz}$ observed frequencies (O'Dea et al. 1991). Like most GPS sources, the $\mathrm{z}=3.529$ QSO OQ172(J1445+0958) shows no kpc-scale radio structure. OQ172 also has an extremely high rest-frame rotation measure ( $\mathrm{RM}>20,000 \mathrm{rad} \mathrm{m}^{-2}$; Udomprasert et al. 1997), one of the highest among $\sim 20$ known high-RM sources. Only 10 mas $\left(R_{\text {proj }} \sim 70 \mathrm{pc}\right)$ from the VLBI core, the RM falls to $<100 \mathrm{rad} \mathrm{m}^{-2}$. This very steep $\mathrm{RM}$ gradient may explain the low polarization $(\chi=\mathrm{P} / \mathrm{I})$ in the core and inner jet, as compared to the higher values of $\chi$ downstream. In order to better understand the circumnuclear environment of OQ172, we performed a VLBA polarimetry observation.

\section{Results and Discussion}

After the data reduction, the linear polarization details would be obtained for every frequencies. When the polarized emission propagates through a magnetized plasma, the polarization plane will rotates with wavelength lamda according to the following expression (e.g. Taylor 1998),

$$
\mathrm{RM}=812 \int_{0}^{\mathrm{L}} \mathrm{n}_{\mathrm{e}} \mathrm{B}_{\|} \mathrm{dl} \operatorname{rad~m}^{-2}
$$

where $\mathrm{RM}$ is the rotation mature in $\mathrm{rad} \mathrm{m}^{-2}, n_{e}$ is the electron number density in $\mathrm{cm}^{-3}$, $B_{\|}$is magnetic field in $\mathrm{mG}$, and the integral is taken over a passage $\mathrm{l}$ in pc alone the line of sight from the observer. In order to examine whether the variation of $\chi$ at different frequency is associated with Faraday rotation, we investigate the relationship between $\chi$ and $\lambda^{2}$ at each frequency. Linear least-squares fit for $\chi$ and $\lambda^{2}$ can be obtained for core and some inner jet region in OQ172 from our observation. Figure 1 presents the masscale Rotation Measure (RM) fit within $5 \& 8 \mathrm{GHz}$ frequencies respectively. Note that when the redshift correction was applied to the wavelengths, the RM values from core component and innermost component in the rest frame of OQ172 are extremely high, 

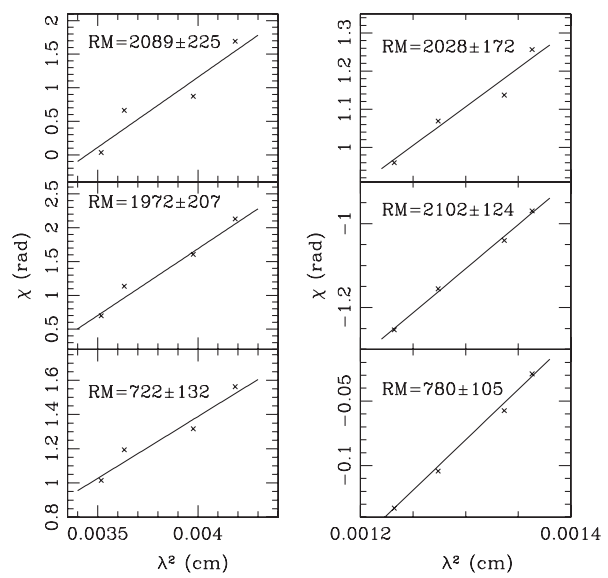

Figure 1. Rotation Measure for GPS quasar OQ172 in core and inner jet part on C and X band.

up to $40000 \mathrm{rad} \mathrm{m}^{-2}$. However, the $\mathrm{RM}$ value goes down very low since a much smaller change in position angel $\chi$ at the outer jet region in OQ172, such as $\mathrm{C} 2$ \& X2 component. It is clear to see that the RM value diminishes along with the jet direction, with a gradient of about several hundreds $\mathrm{rad} \mathrm{m}^{-2} \mathrm{mas}^{-1}$ toward the southwestern jet. From results of our observation, the $\mathrm{RM}$ values at $5 \mathrm{GHz}$ from core region to outer jet part are consistent with the results obtained from Udomprasert et al (1997). At the same time, by using our simultaneous multi-frequency observation, the GPS absorption mechanism of OQ172 would be studied. And the some physical parameters, such as electron density $n_{e}$, magnetic filed $\mathrm{B}_{\|}$, were discussed under each absorption mechanism. A further evidence for the unusual physical environment in the core of OQ172, the near-infrared spectrum shows a typical $\mathrm{H} \beta$ broad emission line FWHM of $\sim 3,700 \mathrm{~km} \mathrm{~s}^{-1}$, but an unusually large width of $2,200 \mathrm{~km} \mathrm{~s}^{-1}$ for the narrow [OIII] $5007 \AA$ line (Hirst et al. 2003). Assuming that Narrow Line Region (NLR) gas dynamics is mainly governed by the inner nuclear bulge gravitational potential (e.g., Nelson \& Whittle 1996), this observed narrow line width is abnormally larger than the value calculated by $\mathrm{M}_{\mathrm{BH}}-\sigma_{[\mathrm{OIII}]}$ relationship in AGNs $\left(\mathrm{M}_{\mathrm{BH}} \sim 3.98 \times 10^{9} \mathrm{M}_{\odot}, \mathrm{Wu} 2009\right)$, which suggests a large mass within the NLR, and/or strong interactions between the emerging jet and dense material therein.

\section{Acknowledgements}

We are grateful to Dr. Gurvits, L. and Dr. Fletcher, A. B. for helpful discussions and suggestion. This work is supported by NSFC under grants 10803015, 11073039, 10978009 and supported by the Ministry of Science and Technology of China (Grant No. 2009CB824900 and 2009CB824800).

\section{References}

Hirst, P., Jackson, N., \& Rawlings, S. 2003, MNRAS, 346, 1009

Nelson, C. H. \& Whittle, M. 1996, ApJ, 465, 96

O'Dea, C. P., Baum S. A., \& Stanghellini C. 1991, ApJ, 380, 66

Taylor, G. B. 1998, ApJ, 506, 637

Udomprasert, P. S., Taylor, G. B., Pearson, T. J., \& Roberts, D. H. 1997, ApJL, 483, L9

Wu, Q. W. 2009, MNRAS, 398, 1905 